Article

\title{
Pulsed Electric Fields (PEF) to Mitigate Emerging Mycotoxins in Juices and Smoothies
}

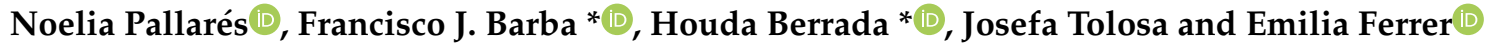 \\ Laboratory of Toxicology and Food Chemistry, Faculty of Pharmacy, University of Valencia, \\ 46100 Burjassot, Valencia, Spain; Noelia.pallares@uv.es (N.P.); Josefa.tolosa@uv.es (J.T.); emilia.ferrer@uv.es (E.F.) \\ * Correspondence: francisco.barba@uv.es (F.J.B.); Houda.berrada@uv.es (H.B.); \\ Tel.: +34-963544972 (F.J.B.); +34-963544117 (H.B.)
}

Received: 8 September 2020; Accepted: 3 October 2020; Published: 7 October 2020

Featured Application: Pulsed electric fields (PEF) technology is explored here as an effective tool for inactivating emerging mycotoxins at low temperatures and over short time periods.

\begin{abstract}
The development of innovative food processing technologies has increased to answer the growing demand to supply of fresh-like products. The aim of the present study is to investigate the effect of pulsed electric fields (PEF) technology on reducing the emerging mycotoxins (enniatins (ENs) and beauvericin (BEA)) contents in juice and smoothie samples. The products of degradation obtained after PEF treatment were identified and their toxicological endpoint toxicities predicted by Pro Tox-II web. Mycotoxin reduction ranged from 43 to $70 \%$ in juices and smoothies, but in water the expected effect was lower. The acidified $\mathrm{pH}$ increased BEA reduction in water. The degradation products that were produced were the result of the loss of aminoacidic fragments of the original molecules, such as HyLv, Val, Ile, or Phe. Pro Tox-II server assigned a toxicity class I for enniatin B (ENB) degradation products with a predicted LD50 of $3 \mathrm{mg} / \mathrm{Kgbw}$. The other degradation products were classified in toxicity class III and IV.
\end{abstract}

Keywords: enniatins; beauvericin; PEF; degradation compounds; DLLME; LC-MS/MS-IT; LC-ESI-qTOF-MS; Pro Tox-II

\section{Introduction}

Excellent nutritional and sensory food properties as well as food security are some of the main population concerns leading to an increased demand of high quality fresh-like foods [1]. High hydrostatic pressure (HPP), pulsed electric field (PEF), and ultrasound (USN), collectively called innovative food processing technologies, are being employed to obtain safe, healthy processed food with, if possible, unaltered sensory and nutritional properties [2].

These technologies are based on physical constraints and offer some advantages compared to traditional thermal processing as they use mild temperatures and short treatment times, thus being a useful alternative to partially or completely replace the traditional well-established processes of preservation [3,4]. PEF technology involves the application of electrical treatments of different electric field strength $(1-40 \mathrm{kV} / \mathrm{cm})$ for short periods of time to a product placed between two electrodes. PEF is reported to be an effective tool for inactivating microorganisms at low temperatures and has been widely used to sterilize foods such as vegetables, fruit juices, milk, and liquid eggs [5,6]. PEF has also been applied to inactivate enzymes in the food industry, to extract nutritionally valuable compounds from plant tissue and food by-products, for drying and freezing in food processing, and to promote some selected properties of food macromolecules and some chemical reactions [7]. Compared with thermal treatments, PEF-processed juices allowed for more retention of biologically active compounds 
such as vitamins, ascorbic acid, carotenoid, anthocyanins, lycopene, and organoleptic characteristics than that of the juices processed under thermal treatment [8]. In this sense, Guo et al. [9] observed that PEF processing did not alter the contents of total phenolics and anthocyanin in pomegranate juice. PEF treatment has also been successfully applied in the degradation of some organophosphorus pesticides [10,11].

Mycotoxins are toxic substances naturally present in food and feed, produced by secondary metabolism of some filamentous fungi [12]. They are related to some adverse effects such as nephrotoxicity, hepatotoxicity, carcinogenicity, mutagenicity, and immunosuppressive effects. Emerging mycotoxins are mycotoxins produced by the Fusarium genus that cause cytotoxic effects due their ionophoric properties, which evoke changes in the intracellular ion concentrations that consequently affect cell functions [13,14].

Development of detoxification technologies should be a priority for research in food processing to reduce mycotoxin levels [15]. However, few studies are available so far in the literature dealing with the effects of PEF on aflatoxins (AFs) content [16,17].

In addition, during food processing, some degradation or modified mycotoxins products may also be formed. A large majority of these compounds that are generated after food processing have not been tested for potential adverse effects on human health, which makes it difficult to carry out an adequate risk assessment [18]. In this case, computational approaches can be used as a preliminary tool to identify the potential toxicity of these degraded or modified mycotoxins compounds generated after food processing by using the compounds' chemical structures, and these approaches may also be employed as a screening method to select which compounds will be assayed later by in vitro assays.

In silico methods are currently one of the promising approaches for toxicity assessment; they employ expert systems and multiple algorithms that use computation [19] and are efficient tools, especially in concrete situations, such as evaluating the toxicity of new degradation or reaction products for which standards are not available. Furthermore, these in silico methods lower costs, duration, and harm to animals, thus, complying with the replace, reuse, and refinement of experimental animals (3Rs) protocol [20].

In this context, the aim of the present work was to study the effect of PEF technology on emerging mycotoxins contents (enniatin A (ENA), enniatin A1 (ENA1), enniatin B (ENB), enniatin B1 (ENB1) and beauvericin (BEA)) in grape juices and smoothies. After the PEF treatment, enniatins (ENs) and BEA were extracted by dispersive liquid-liquid microextraction (DLLME) and determined by HPLC-MS/MS-IT. In addition, HPLC-Q-TOF-MS was used for tentative identification of ENs and BEA degradation products. Finally, different toxicological endpoints were predicted by the ProTox-II web server's in silico method in order to evaluate and compare the toxicity of the identified degradation products with the precursor mycotoxin.

\section{Materials and Methods}

\subsection{Reagents and Chemicals}

Acetonitrile (ACN), methanol (MeOH) (HPLC grade) and chloroform $\left(\mathrm{CHCl}_{3}\right)(99 \%$ grade) used for the extraction were purchased from Merck (Darmstadt, Germany). Ethyl acetate (EtOAc) (HPLC grade $99.5+\%$ ) was supplied by Alfa Aesar (Karlsruhe, Germany). The deionized water employed for the mobile phase with resistivity $>18 \mathrm{M} \Omega \mathrm{cm}$ was prepared using a Milli-Q SP${ }^{\circledR}$ Reagent Water System (Millipore Corporation, Bedford, USA). All solvents employed to prepare mobile phases were filtered prior to use through a $0.45 \mu \mathrm{m}$ cellulose filter supplied by Scharlau (Barcelona, Spain).

The salt, ammonium formate (99\%), was obtained from Panreac Quimica S.A.U. (Barcelona, Spain). Formic acid (reagent grade $\geq 95 \%$ ) was purchased from Sigma-Aldrich (St. Louis, MO, USA). Sodium chloride $(\mathrm{NaCl})$ was supplied by VWR Chemicals (Leuven, Belgium). All samples were filtered by a $13 \mathrm{~mm} / 0.22 \mu \mathrm{m}$ nylon filter acquired from Membrane Solutions (TX, USA) prior to injection. 
Standards of the mycotoxins ENA, ENA1, ENB, ENB1, and BEA were supplied by Sigma (St. Louis, MO, USA) and prepared in methanol at concentration of $1000 \mathrm{mg} / \mathrm{L}$. The appropriate working solutions were prepared from the stock solutions. All solutions were placed at $-20^{\circ} \mathrm{C}$ until the analysis.

\subsection{Samples}

For this study, grape juice and smoothie samples were employed. Smoothie samples were made from apple, banana, grape juices, strawberry, blackberry, orange purees, and cereals. Ten bottles of juice and smoothie samples were purchased from different supermarkets in Valencia. The $\mathrm{pH}$ values of fruit juices and smoothies were measured using a $\mathrm{pH}$ meter before the study, registering values from 3.8 to 4 . After the homogenization of the beverages, aliquots were taken, and samples were tested for the absence of mycotoxins. After this, volumes of $215 \mathrm{~mL}$ were spiked individually with ENA, ENA1, ENB, ENB1, and BEA, respectively, at concentration of $100 \mu \mathrm{g} / \mathrm{L}$. The same experiments were performed in parallel in water samples spiked with each studied mycotoxin at concentrations of $100 \mu \mathrm{g} / \mathrm{L}$. All experiments were performed in triplicate.

\subsection{Pulsed Electric Field Treatment}

For the PEF treatment, the PEF-Cellcrack III (German Institute of Food Technologies (DIL)) equipment (ELEA, Quakenbrück, Osnabrück, Germany), with a treatment chamber with a $10 \mathrm{~cm}$ space between electrodes, was used. The voltage was set at $30 \mathrm{kV}$, resulting in a field strength of $3 \mathrm{kV} / \mathrm{cm}$, and the specific energy applied was $500 \mathrm{~kJ} / \mathrm{kg}$. To reach the $500 \mathrm{~kJ} / \mathrm{kg}$, an average of 238 pulses were applied in different cycles, during approximately $5 \mathrm{~min}$. During the treatment, the temperature did not exceed $75^{\circ} \mathrm{C}$ with conductivity of $2890 \mu \mathrm{s} / \mathrm{cm}$ for grape juice and $3160 \mu \mathrm{s} / \mathrm{cm}$ for smoothies.

\subsection{Dispersive Liquid-Liquid Microextraction Procedure (DLLME)}

The samples were extracted according to the method proposed in a previous work [21]. Five milliliters of sample were placed in a $10 \mathrm{~mL}$ conical tub with $1 \mathrm{~g}$ of $\mathrm{NaCl}$ and shaken for one minute in vortex. Then, a mixture of dispersant and extractant solvents ( $950 \mu \mathrm{L}$ of ACN and $620 \mu \mathrm{L}$ of EtOAc) were added, and shaken for one minute, resulting in a cloudy solution of the three components. The mixture was centrifuged at $4000 \mathrm{rpm}$ for $5 \mathrm{~min}$, allowing for the separation of phases. The organic phase was separated and placed into other conical tubes. The mixture of dispersant and extractant solvents $\left(950 \mu \mathrm{L}\right.$ of $\mathrm{MeOH}$ and $620 \mu \mathrm{L}$ of $\left.\mathrm{CHCl}_{3}\right)$ was added to the remaining residue. After shaking and centrifugation, the organic phase was separated and placed with the first organic phase. The organic phases were evaporated to near dryness under a nitrogen stream using a Turvovap LV Evaporator (Zymark, Hoptikinton, USA). The residue obtained was reconstituted in a vial with $1 \mathrm{~mL}$ of $20 \mathrm{mM}$ ammonium formate $(\mathrm{MeOH} / \mathrm{ACN})(50 / 50 \mathrm{v} / \mathrm{v})$ and filtered through a $13 \mathrm{~mm} / 0.22 \mu \mathrm{m}$ nylon filter prior to the injection in LC-MS/MS-IT.

\subsection{LC-MS/MS-IT Determination}

To determine the mycotoxins contents, an Agilent 1200 chromatograph (Agilent Technologies, Palo Alto, CA, USA) equipped with 3200 QTRAP ${ }^{\circledR}$ (Applied Biosystems, AB Sciex, Foster City, CA, USA) with Turbo Ion Spray (ESI) electrospray ionization was used. The QTRAP analyzer combines a fully functional triple quadrupole and a linear ion trap mass spectrometer. The chromatographic separation of analytes was performed using a Gemini-NX column C18 (Phenomenex, $150 \mathrm{~mm} \times 4.6 \mathrm{~mm}$, $5 \mu \mathrm{m}$ particle size) preceded by a guard column. Mobile phases were mobile phase A ( $5 \mathrm{mM}$ ammonium formate, $0.1 \%$ formic acid water) and mobile phase $\mathrm{B}(5 \mathrm{mM}$ ammonium formate, $0.1 \%$ formic acid methanol). The gradient program initiated with a proportion of $0 \%$ for eluent $B$; in 10 min it increased to $100 \%$, then decreased to $80 \%$ in $5 \mathrm{~min}$, and finally decreased to $70 \%$ in $2 \mathrm{~min}$. In the next $6 \mathrm{~min}$, the column was cleaned, readjusted to initial conditions, and equilibrated for $7 \mathrm{~min}$. The flow rate was fixed at $0.25 \mathrm{~mL} / \mathrm{min}$, the injection volume was $20 \mu \mathrm{L}$, and the oven temperature was set at $40^{\circ} \mathrm{C}$. 
The Turbo Ion Spray operated in positive ionization mode (ESI+) for the analysis. Nitrogen served as the nebulizer and collision gas. During the analysis, the following parameters were fixed: ion spray voltage, $5500 \mathrm{~V}$; curtain gas, 20 arbitrary units; GS1 and GS2, 50 and 50 psi, respectively; probe temperature (TEM), $450{ }^{\circ} \mathrm{C}$.

\subsection{LC-ESI-qTOF-MS Analysis}

For the identification of degradation products, an Agilent 1200-LC system (Agilent Technologies, Palo Alto, CA, USA), for the chromatographic determination, coupled to a 6540 Agilent UltraHigh-Definition Accurate-Mass q-TOF-MS, equipped with an Agilent Dual Jet Stream electrospray ionization (Dual AJS ESI) for mass spectrometry analysis, was employed. Chromatographic separation was developed in a Gemini-NX column C18 (Phenomenex, $150 \mathrm{~mm} \times 4.6 \mathrm{~mm}, 5 \mu \mathrm{m}$ particle size). The mobile phases consisted of water (A) and acetonitrile (B), both with $0.1 \%$ of formic acid. The gradient was as follows: $0-6 \mathrm{~min}, 50 \% \mathrm{~B} ; 7-12 \mathrm{~min}, 100 \% \mathrm{~B} ; 13-20 \mathrm{~min}, 50 \%$ B. The injection volume was $5 \mu \mathrm{L}$ and the flow rate $0.2 \mathrm{~mL} / \mathrm{min}$. The following conditions were employed for mass spectrometry: interface in positive ionization mode; drying gas flow $\left(\mathrm{N}_{2}\right), 12.0 \mathrm{~L} \mathrm{~min}^{-1}$; nebulizer pressure, $50 \mathrm{psi}$; gas drying temperature, $370{ }^{\circ} \mathrm{C}$; capillary voltage, $3500 \mathrm{~V}$; fragmentor voltage, $160 \mathrm{~V}$. Analyses were carried out in MS mode, and MS spectra were collected within the scan range 50-1500 m/z.

\subsection{Method Validation}

Our method was previously validated in the laboratory for emerging mycotoxins analysis in juices [21]. For validation, the method was characterized in terms of recovery, repeatability (intraday precision), reproducibility (interday precision), matrix effects, limit of detection (LOD), and limit of quantification (LOQ) according to the Commission Decision [22]. The recoveries performed at three levels of contamination $(50,100$, and $200 \mu \mathrm{g} / \mathrm{L}$ ) ranged from 66 to $112 \%$. Intraday precision and interday precision were lower than $14 \%$ and $19 \%$, respectively. LODs were between 0.15 and $1.5 \mu \mathrm{g} / \mathrm{L}$ and LOQs were between $0.5 \mu \mathrm{g} / \mathrm{L}$ and $5 \mu \mathrm{g} / \mathrm{L}$, respectively. Matrix effects experiments revealed signal suppression from 52 to $73 \%$. Regarding linearity, regression coefficients were higher than 0.990 in all cases.

\subsection{In Silico Prediction Methods}

The ProTox-II (http://tox.charite.de/protox_II/) is a free web server to predict diverse toxicological endpoints for several chemical compounds [23,24]. This tool incorporates molecular similarity, pharmacophores, fragment propensities, and machine-learning models for the prediction of some toxicity endpoints, such as acute toxicity, hepatotoxicity, cytotoxicity, carcinogenicity, mutagenicity, immunotoxicity, adverse outcomes pathways (Tox21), and toxicity targets, representing a novel approach in toxicity prediction [24].

The ProTox-II platform is divided into a five different classification steps constructed by different computational models: (1) acute toxicity (oral toxicity model with five different toxicity classes); (2) organ toxicity model (1 model); (3) toxicological and genotoxicological endpoints, mainly immunotoxicity, cytotoxicity, mutagenicity, and carcinogenicity (4 models); (4) toxicological pathways (12 models); and (5) toxicity targets (15 models).

Regarding the oral acute toxicity, toxic doses are expressed as LD50 values in $\mathrm{mg} / \mathrm{Kgbw}$. The prediction accuracy derived from cross-validation results is also given. Depending on the LD50 for each substance, the substances can be classified into different toxicity classes, which are defined according to the Globally Harmonized System (GHS) of classification in labelling of chemicals [25]:

- Class I: fatal if swallowed (LD50 $\leq 5 \mathrm{mg} / \mathrm{Kgbw}$ )

- Class II: fatal if swallowed ( $5 \mathrm{mg} / \mathrm{kg}<\mathrm{LD} 50 \leq 50 \mathrm{mg} / \mathrm{Kgbw})$

- Class III: toxic if swallowed (50 mg/kg $<$ LD50 $\leq 300 \mathrm{mg} / \mathrm{Kgbw})$

- Class IV: harmful if swallowed (300 mg/kg < LD50 $\leq 2000 \mathrm{mg} / \mathrm{Kgbw})$

- Class V: may be harmful if swallowed $(2000 \mathrm{mg} / \mathrm{kg}<\mathrm{LD} 50 \leq 5000 \mathrm{mg} / \mathrm{Kgbw})$ 
Regarding the toxicity endpoint and organ toxicity prediction, the predictive models are based on data from both in vitro (e.g., Tox21 assays, Ames bacterial mutation assays, hepG2 cytotoxicity assays, immunotoxicity assays, among others) and in vivo assays (e.g., carcinogenicity, hepatotoxicity).

In ProTox-II, two types of target-pathway-based models can be found. The two pathways have been defined as (i) Nuclear Receptor Signaling Pathways (7 pathway assays shown in Table 4) and (ii) Stress Response Pathways (5 pathway assays shown in Table 5).

This approach is based on the fact that a chemical compound can activate or inhibit a receptor or an enzyme when they interact, resulting in perturbation of diverse biological pathways and disruption of cellular processes, thereby leading to cell death. The main purpose of the initiative was to prioritize substances for further in-depth toxicological evaluation as well as to identify some mechanisms for further investigation such as disease-associated pathways. By applying this computational prediction tool, it is possible to test quickly and efficiently whether certain chemical compounds have the potential to disrupt processes in the human body that may lead to adverse health effects [24].

\section{Results and Discussion}

\subsection{Reduction of ENs and BEA Contents after PEF Treatment}

After PEF treatment, a significant reduction of ENA, ENA1, ENB, ENB1, and BEA was observed in all tested samples. For juice samples, the contents of emerging mycotoxins obtained were $46.81 \pm 0.8 \mu \mathrm{g} / \mathrm{L}$ (ENA), $52.23 \pm 4.4 \mu \mathrm{g} / \mathrm{L}$ (ENA1), $56.98 \pm 10.88 \mu \mathrm{g} / \mathrm{L}$ (ENB), $56.8 \pm 2.79 \mu \mathrm{g} / \mathrm{L}$ (ENB1), and $54.04 \pm 0.4 \mu \mathrm{g} / \mathrm{L}$ (BEA), corresponding to reduction percentages from 43 to $53 \%$, approximately (Figure 1 ).

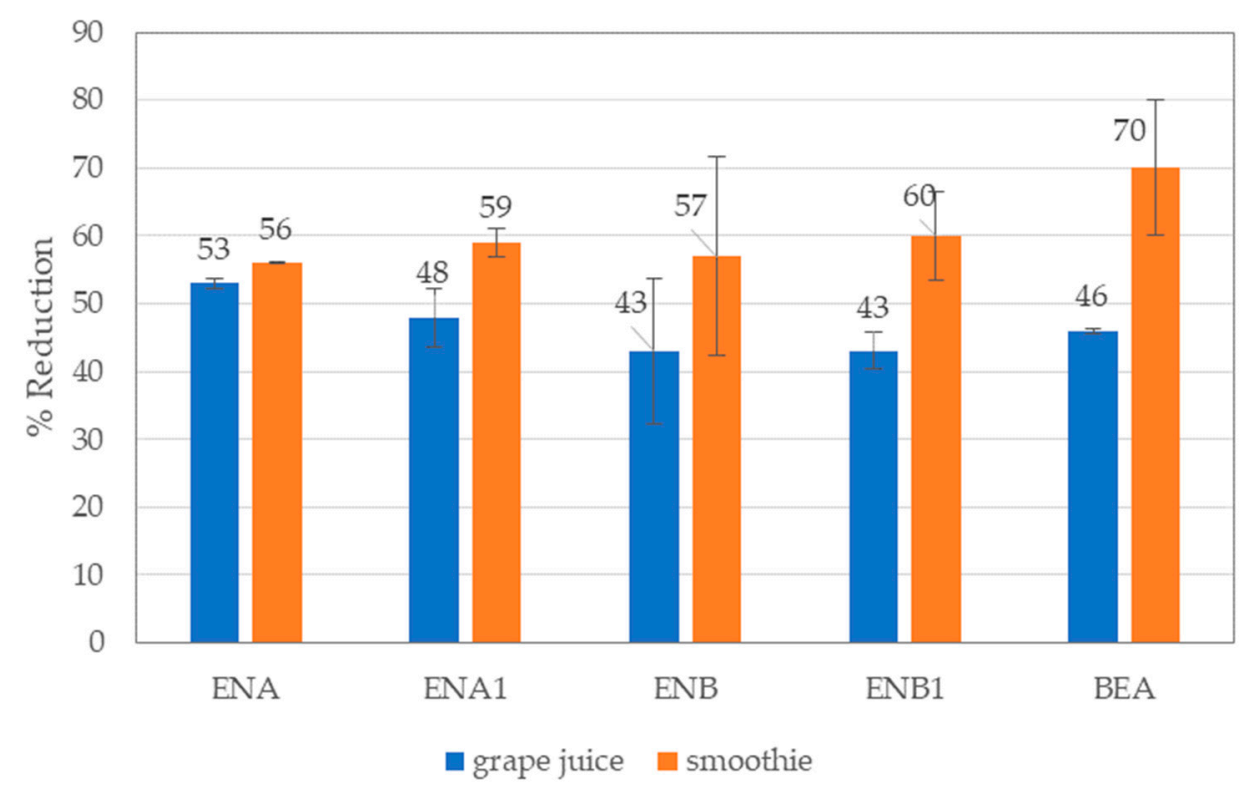

Figure 1. Percentage of reduction (\%) of enniatins (ENs) and beauvericin (BEA) in grape juice vs. smoothie samples after pulsed electric fields (PEF) treatment.

For smoothie samples, the contents after PEF treatment were $44.35 \pm 0.21 \mu \mathrm{g} / \mathrm{L}$ (ENA), $40.84 \pm 2.12 \mu \mathrm{g} / \mathrm{L}$ (ENA1), $42.94 \pm 14.56 \mu \mathrm{g} / \mathrm{L}$ (ENB), $40.12 \pm 6.58 \mu \mathrm{g} / \mathrm{L}$ (ENB1), and $29.8 \pm 9.8 \mu \mathrm{g} / \mathrm{L}$ (BEA), corresponding with higher reduction percentages from 56 to 70\%, approximately (Figure 1). Figure 2 shows the chromatograms of juice samples spiked with ENB1: PEF-treated vs. non-treated samples. 


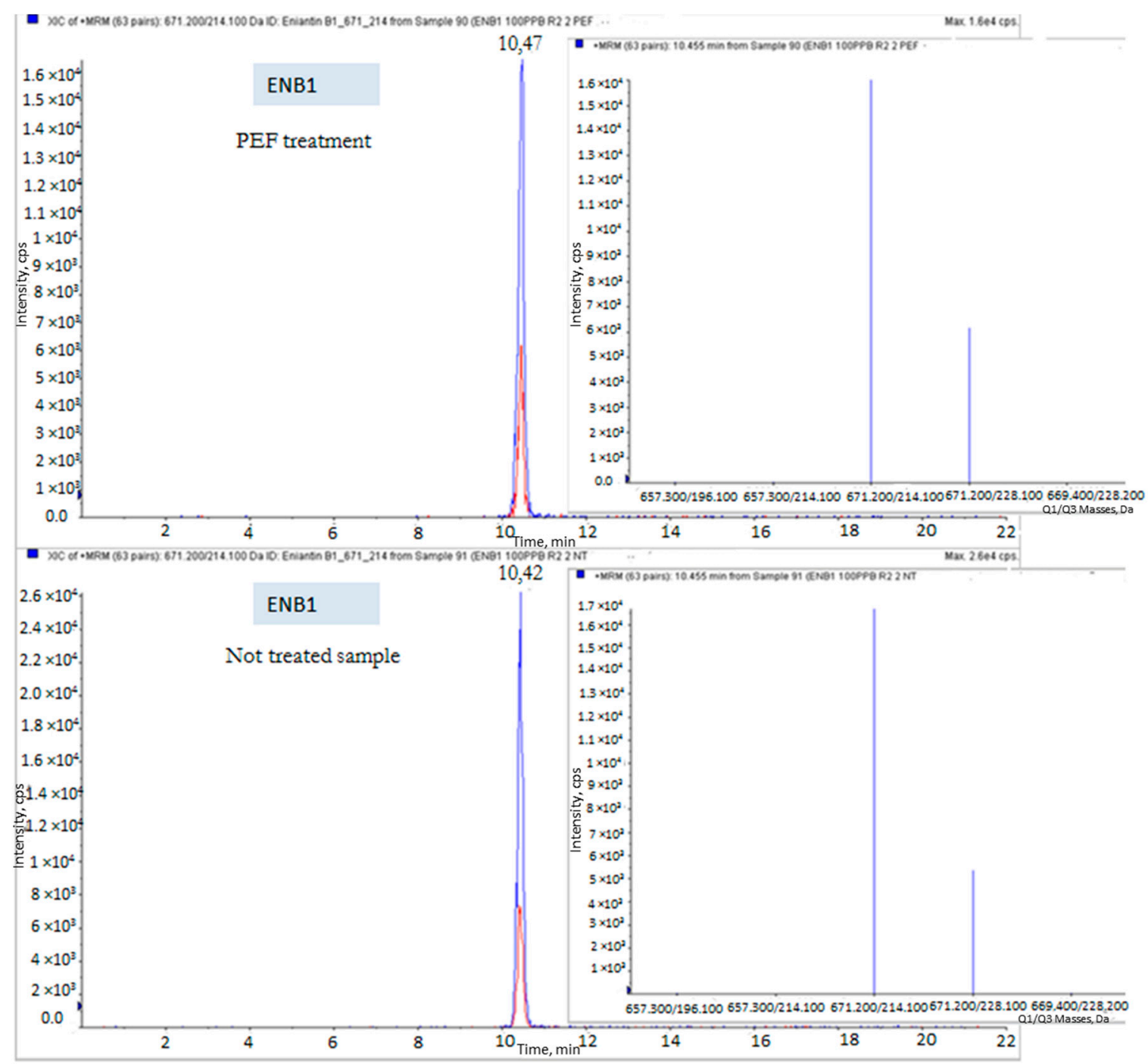

Figure 2. LC-MS/MS-IT chromatogram of juice sample contaminated by enniatin B1 (ENB1) treated by pulsed electric fields (PEF) vs. non-treated.

During PEF treatment, temperatures around $70{ }^{\circ} \mathrm{C}$ were reached. The emerging mycotoxins were relatively sensible to temperature, therefore, experiments in $\mathrm{H}_{2} \mathrm{O}$ spiked with mycotoxins at the same conditions and heated at $70{ }^{\circ} \mathrm{C}$ during the same duration of PEF treatment were performed in order to check if the degradation observed was a consequence of the temperatures reached during the PEF treatment. The results obtained showed that temperature treatment caused degradations percentages of $26 \%$.

In previous studies, Serrano et al. [26] and Tolosa et al. [27] observed similar ENB and ENB1 reductions after thermal treatments of cooking pasta and boiling fish at temperatures $>100{ }^{\circ} \mathrm{C}$, with reduction percentages from 14 to $49 \%$ for ENB and 53 to $65 \%$ for ENB1 after cooking pasta and to nearly $60 \%$ (ENB and ENB1) after boiling fish.

As was mentioned above, scarce information is available in the literature about mycotoxin reduction after the PEF treatment. Vijayalakshmi et al. [16] optimized the combination of thermal processing with PEF to reduce artificially spiked AFs in a potato, dextrose, and agar system, at different $\mathrm{pH}$ values. The combination treatment was found to be more effective than individual processes, with reduction percentages from 92.3 to $96.9 \%$ for AFB1 as a function of $\mathrm{pH}$ and from 82 to $95.7 \%$ for total AFs. These authors, Vijayalakshmi et al. [17], also observed a reduction of AFB1 and AFs contents 
from 77 to $97 \%$ after optimizing PEF methodology. These reductions were similar to those obtained in the present study for BEA in smoothie samples (70\%).

The same treatment applied to $\mathrm{H}_{2} \mathrm{O}$ revealed lower percentages of reduction (from 31 to $45 \%$ ) (Figure 3). The lower percentages of reduction observed in water vs. grape juices and smoothies (43 to $70 \%$ ) may be due to differences in the matrix and/or in $\mathrm{pH}$. The $\mathrm{pH}$ values of the juices and smoothies employed in this study were measured to be 3.88 and 3.94, respectively. The effect of $\mathrm{pH}=4$ was assessed on ENs and BEA contents using a water model acidified with lemon juice. An additional degradation was observed when the $\mathrm{H}_{2} \mathrm{O}$ was acidified for BEA (56\%) (Figure 3) compared with neutral $\mathrm{H}_{2} \mathrm{O}$, but significant differences were not observed for ENs.

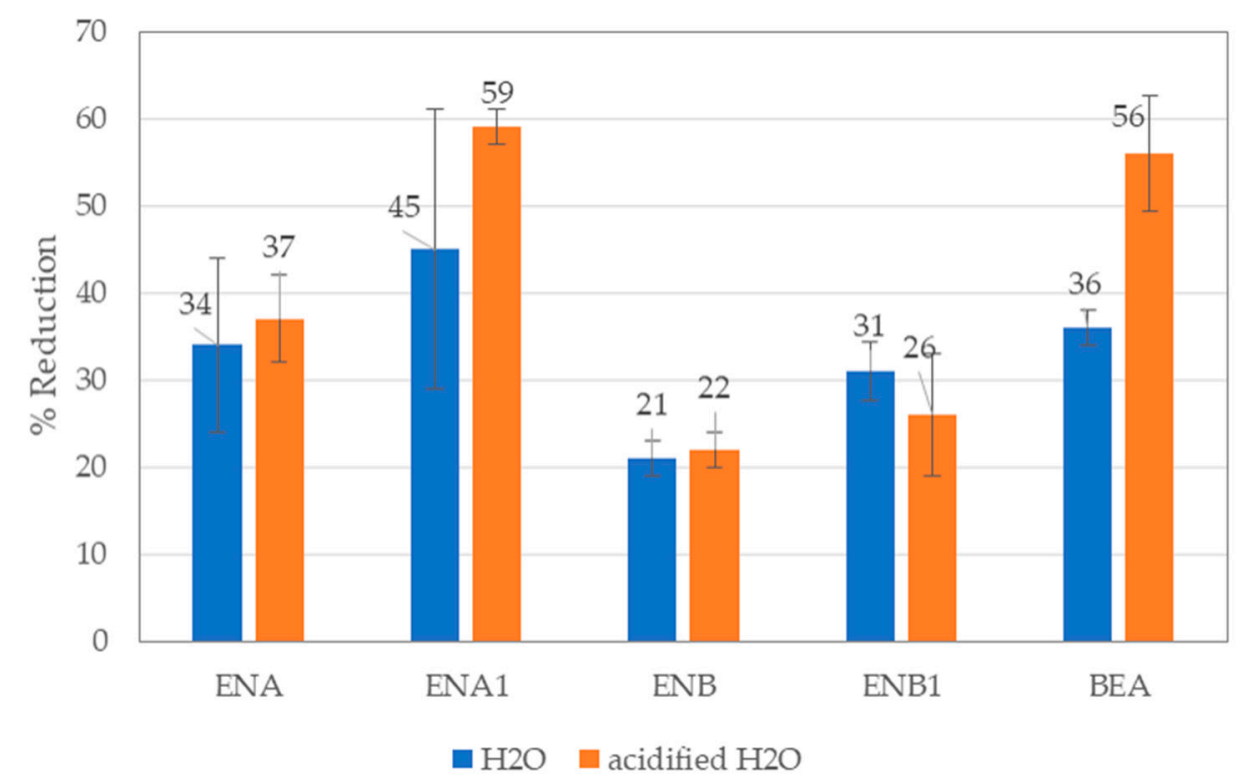

Figure 3. Percentage of reduction (\%) of ENs and $\mathrm{BEA}$ in $\mathrm{H}_{2} \mathrm{O}$ vs. acidified $\mathrm{H}_{2} \mathrm{O}$ at $\mathrm{pH}=4$ after pulsed electric fields (PEF) treatment.

As reported by other authors [28,29], the degradation could be dependent of juice constituents. This fact may explain the different reductions observed in juices compared to the reductions observed in water, and the higher reductions observed in smoothie samples (from 56 to $70 \%$ ) compared to grape juice samples (from 43 to 53\%). Smoothies were made with different fruit juices, purees, and cereals, so they have a more complex matrix than that of grape juice.

\subsection{Identification of Degradation Products}

Several degradation products of ENs and BEA were tentatively identified after PEF treatment in juice and smoothie matrices (Table 1). For this purpose, the samples were injected in LC-ESI-qTOF-MS equipment, operating in the full scan modality. For BEA, the degradation product with $\mathrm{m} / \mathrm{z}$ 517.3705, corresponded to BEA with the loss of one unit of phenylalanine (Phe) and hydroxyvaleric acid (HyLv). In a previous study, Meca et al. [30] also observed a degradation product from BEA with the loss of these two structural components (Phe + HyLv).

For ENA, one degradation product was identified at $\mathrm{m} / \mathrm{z} 475.3261$, which corresponds to the loss of isoleucine (Ile) and HyLv. This degradation product was also previously identified by Serrano et al. [31]. For ENA1, the degradation product $\mathrm{m} / \mathrm{z} 475.3244$, corresponding to the loss of valine (Val) and HyLv, was identified. For ENB, two degradation products were observed. The degradation product 1 ( $\mathrm{m} / \mathrm{z}$ 437.1936), characterized as the sodium adduct of ENB, with the loss of Val and HyLv. The degradation product 2 with $\mathrm{m} / \mathrm{z} 527.2000$ corresponds to the loss of Val and was previously 
reported by Serrano et al. [31]. Finally, for ENB1, the degradation product $\mathrm{m} / \mathrm{z} 443.1674$ was identified as the loss of Val and HyLv (Figure 4).

Table 1. LC-ESI-qTOF-MS data (MS1) of the degradation products obtained in juice and smoothie samples after PEF treatment.

\begin{tabular}{|c|c|c|c|c|}
\hline Mycotoxin & Degradation Product & {$[\mathrm{M}+\mathrm{H}]^{+}(\mathrm{m} / \mathrm{z})$} & Lost Fragment & Molecular Structure \\
\hline $\begin{array}{l}\text { ENA degradation } \\
\text { product }\end{array}$ & 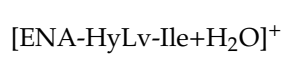 & 475.3261 & HyLV+Ile & \\
\hline $\begin{array}{l}\text { ENA1 degradation } \\
\text { product }\end{array}$ & 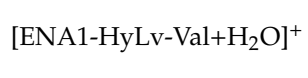 & 475.3244 & HyLv+Val & \\
\hline $\begin{array}{l}\text { ENB degradation } \\
\text { product } 1\end{array}$ & {$[\mathrm{ENB}-\mathrm{HyLv}-\mathrm{Val}+\mathrm{Na}]^{+}$} & 437.1936 & HyLv+Val & \\
\hline $\begin{array}{l}\text { ENB degradation } \\
\text { product } 2\end{array}$ & {$\left[\mathrm{ENB}-\mathrm{Val}+\mathrm{H}_{2} \mathrm{O}\right]^{+}$} & 527.2000 & Val & \\
\hline $\begin{array}{l}\text { ENB1 degradation } \\
\text { product }\end{array}$ & [ENB1-HyLv-Val] $^{+}$ & 443.1674 & HyLv+Val & \\
\hline $\begin{array}{l}\text { BEA } \\
\text { degradation } \\
\text { product }\end{array}$ & {$\left[\text { BEA-Phe-HyLv }+2 \mathrm{H}_{2} \mathrm{O}\right]^{+}$} & 517.3705 & Phe+HyLv & \\
\hline
\end{tabular}




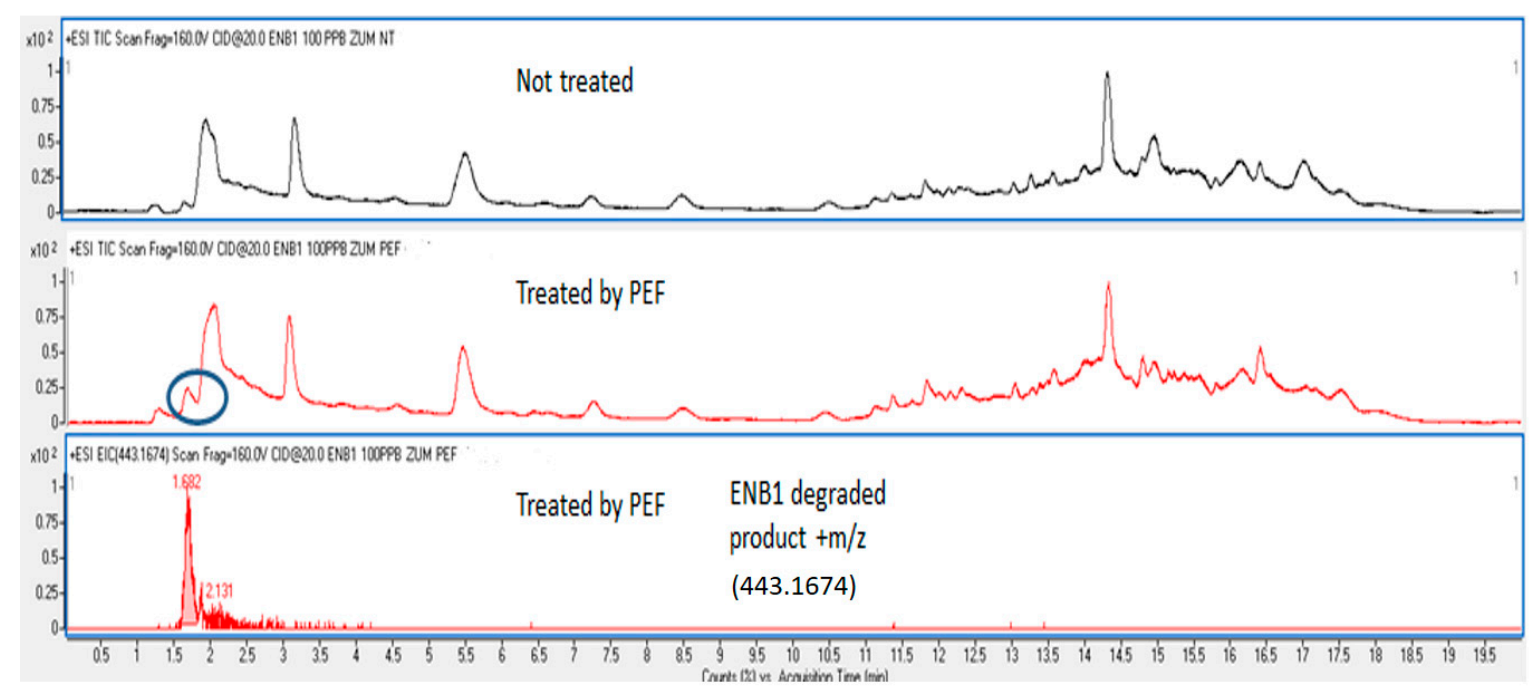

Figure 4. LC-ESI-qTOF-MS chromatogram of degraded product ENB1 m/z 443.1674 obtained in matrix samples after PEF treatment.

The degradation products that were observed confirmed the reduction effect of PEF on emerging mycotoxins. The degradation products originated from the loss of structural aminoacidic fragments of original molecules such as HyLv, Val, Ile, or Phe.

\subsection{In Silico Prediction Methods}

In silico prediction methods were used in this work to evaluate the toxicity of detected and identified degradation products by using the ProTox-II web server. Results of oral acute toxicity expressed as LD50 (mg/Kgbw) and the corresponding toxicity class for each identified compound are shown in Table 2. It should be highlighted that, according to the obtained predictions, both ENB degradation products showed a predicted LD50 of $3 \mathrm{mg} / \mathrm{Kgbw}$, both with $100 \%$ average similarity and prediction accuracy. Thus, the assigned toxicity class was I. According to this result, special attention should be paid to those degradation products, as their predicted toxicity is comparable to that of ENB and also T-2 Toxin (Table 2), which is known to be a toxic fungal metabolite with the lowest tolerable daily intake (TDI) within the Fusarium mycotoxins [32].

Table 2. Acute oral toxicity prediction obtained by using ProTox-II web server.

\begin{tabular}{ccccc}
\hline \multirow{2}{*}{ Mycotoxin } & \multicolumn{4}{c}{ Oral Toxicity Prediction Results } \\
\cline { 2 - 5 } & $\begin{array}{c}\text { Predicted LD50 } \\
\mathbf{( m g / K g b w )}\end{array}$ & $\begin{array}{c}\text { Predicted } \\
\text { Toxicity Class }\end{array}$ & $\begin{array}{c}\text { Average } \\
\text { Similarity (\%) }\end{array}$ & $\begin{array}{c}\text { Prediction } \\
\text { Accuracy (\%) }\end{array}$ \\
\hline ENA degradation product & 1600 & IV & 76.28 & 69.26 \\
ENA1 degradation product & 1600 & IV & 76.28 & 69.26 \\
ENB degradation product & 3 & I & 100 & 100 \\
ENB degradation product (2) & 3 & I & 100 & 100 \\
ENB1 degradation product & 1600 & IV & 75.91 & 69.26 \\
BEA degradation product & 200 & III & 75.89 & 69.26 \\
\hline
\end{tabular}

Class I: fatal if swallowed (LD50 $\leq 5 \mathrm{mg} / \mathrm{Kgbw})$; Class II: fatal if swallowed ( $5 \mathrm{mg} / \mathrm{Kgbw}<\mathrm{LD} 50 \leq 50 \mathrm{mg} / \mathrm{Kgbw})$; Class III: toxic if swallowed ( $50 \mathrm{mg} / \mathrm{Kgbw}<\mathrm{LD} 50 \leq 300 \mathrm{mg} / \mathrm{Kgbw})$; Class IV: harmful if swallowed (300 mg/Kgbw $<$ LD50 $\leq 2000 \mathrm{mg} / \mathrm{Kgbw})$; Class V: may be harmful if swallowed $(2000 \mathrm{mg} / \mathrm{Kgbw}<\mathrm{LD} 50 \leq 5000 \mathrm{mg} / \mathrm{Kgbw})$.

Using the ProTox-II web server, the organ toxicity can be also predicted, specifically the hepatotoxicity, which has been evaluated for different identified compounds, as the liver is the organ where mycotoxins are metabolized. The results obtained regarding the organ toxicity and also the calculated predictions for diverse toxicological endpoints using the ProTox-II web server are 
reported in Table 3. These results showed that identified degradation products were predicted as inactive compounds for hepatotoxicity. However, the ENB1 degradation product was predicted as an active compound for an immunotoxicity endpoint, although the percentage of prediction accuracy (probability score) was low (51\%).

Table 3. Organ toxicity and toxicological endpoint predictions calculated using the ProTox-II web server.

\begin{tabular}{|c|c|c|c|c|c|}
\hline \multirow{3}{*}{$\begin{array}{c}\text { Mycotoxin } \\
\text { (Degradation Product) }\end{array}$} & \multicolumn{5}{|c|}{ Classification } \\
\hline & \multirow{2}{*}{$\begin{array}{c}\begin{array}{c}\text { Organ Toxicity } \\
\text { (\% Probability) }\end{array} \\
\text { Hepatotoxicity }\end{array}$} & \multicolumn{4}{|c|}{$\begin{array}{l}\text { Toxicity Endpoint } \\
\text { (\% Probability) }\end{array}$} \\
\hline & & Carcinogenicity & Immunotoxicity & Mutagenicity & Cytotoxicity \\
\hline ENA & Inactive (74) & Inactive (56) & Inactive (83) & Inactive (68) & Inactive (63) \\
\hline ENA1 & Inactive (74) & Inactive (56) & Inactive (83) & Inactive (68) & Inactive (63) \\
\hline ENB & Inactive (76) & Inactive (58) & Inactive (77) & Inactive (68) & Inactive (58) \\
\hline${ }^{*}$ ENB & Inactive (76) & Inactive (58) & Inactive (89) & Inactive (77) & Inactive (64) \\
\hline ENB1 & Inactive (75) & Inactive (56) & Active (51) & Inactive (76) & Inactive (66) \\
\hline BEA & Inactive (85) & Inactive (53) & Inactive (91) & Inactive (76) & Inactive (70) \\
\hline
\end{tabular}

The prediction results obtained for the toxicological pathways, nuclear receptor signaling pathways, and stress response pathways are reported in Tables 4 and 5, respectively. According to the Tox 21 Consortium, chemical compounds might have the potential to disrupt processes in the human body, which may lead to negative health effects [23]. Regarding the nuclear receptor signaling pathway, seven different pathways were assessed while for the stress response pathways, five diverse assays were evaluated. The computational estimations revealed that the degradation products identified in the present study were predicted as inactive for all of the analyzed pathways. However, this computational analysis is presented only as a useful support tool for the exploration of mitigation strategies; more detailed toxicological studies must be carried out to confirm the reduction of toxicity of the PEF mycotoxins degradation products obtained.

Table 4. Toxicological pathways: Nuclear receptor signaling pathways predicted for detected mycotoxins.

\begin{tabular}{|c|c|c|c|c|c|c|c|}
\hline \multirow[b]{2}{*}{$\begin{array}{c}\text { Mycotoxin } \\
\text { (Degradation } \\
\text { Product) }\end{array}$} & \multicolumn{7}{|c|}{ Tox21 Nuclear Receptor Signaling Pathways (\% Probability) } \\
\hline & $\begin{array}{c}\text { Aryl } \\
\text { Hydrocarbon } \\
\text { Receptor } \\
\text { (AhR) }\end{array}$ & $\begin{array}{c}\text { Androgen } \\
\text { Receptor (AR) }\end{array}$ & $\begin{array}{c}\text { Androgen } \\
\text { Receptor Ligand } \\
\text { Binding Domain } \\
\text { (AR-LBD) }\end{array}$ & Aromatase & $\begin{array}{c}\text { Estrogen } \\
\text { Receptor } \\
\text { Alpha (ER) }\end{array}$ & $\begin{array}{c}\text { Estrogen } \\
\text { Receptor Ligand } \\
\text { Binding Domain } \\
\text { (ER-LBD) }\end{array}$ & $\begin{array}{c}\text { Peroxisome } \\
\text { Proliferator Activated } \\
\text { Receptor Gamma } \\
\text { (PPAR-Gamma) }\end{array}$ \\
\hline ENA & Inactive (97) & Inactive (95) & Inactive (97) & Inactive (98) & Inactive (87) & Inactive (97) & Inactive (98) \\
\hline ENA1 & Inactive (97) & Inactive (95) & Inactive (97) & Inactive (98) & Inactive (87) & Inactive (97) & Inactive (98) \\
\hline ENB & Inactive (98) & Inactive (97) & Inactive (99) & Inactive (98) & Inactive (83) & Inactive (96) & Inactive (98) \\
\hline BEA & Inactive (93) & Inactive (95) & Inactive (98) & Inactive (95) & Inactive (89) & Inactive (97) & Inactive (95) \\
\hline
\end{tabular}

* Degradation product (2).

Table 5. Toxicological pathways: Stress response pathways predicted for detected mycotoxins.

\begin{tabular}{lccccc}
\hline $\begin{array}{c}\text { Mycotoxin } \\
\text { (Degradation } \\
\text { Product) }\end{array}$ & $\begin{array}{c}\text { Nuclear Factor } \\
\text { (Erythroid-Derived } \\
\text { 2-Like 2/Antioxidant } \\
\text { Responsive Element) } \\
\text { (nrf2/ARE) }\end{array}$ & $\begin{array}{c}\text { Heat Shock } \\
\text { Factor } \\
\text { Response } \\
\text { Element (HSE) }\end{array}$ & $\begin{array}{c}\text { Mitochondrial } \\
\text { Membrane } \\
\text { Potential } \\
\text { (MMP) }\end{array}$ & $\begin{array}{c}\text { Phosphoprotein } \\
\text { (Tumor Suppressor) } \\
\text { p53 }\end{array}$ & $\begin{array}{c}\text { ATPase Family AAA } \\
\text { Domain Containing } \\
\text { Protein 5 (ATAD5) }\end{array}$ \\
\hline ENA & Inactive (98) & Inactive (98) & Inactive (98) & Inactive (98) & Inactive (98) \\
ENA1 & Inactive (98) & Inactive (98) & Inactive (98) & Inactive (98) & Inactive (98) \\
ENB & Inactive (99) & Inactive (99) & Inactive (98) & Inactive (99) & Inactive (97) \\
*ENB & Inactive (99) & Inactive (99) & Inactive (99) & Inactive (99) & Inactive (96) \\
ENB1 & Inactive (98) & Inactive (98) & Inactive (97) & Inactive (97) & Inactive (99) \\
BEA & Inactive (97) & Inactive (97) & Inactive (91) & Inactive (96) & Inactive (95) \\
\hline
\end{tabular}

* Degradation product (2). 
The obtained probability scores in Tables 4 and 5 showed an adequate accuracy in predicted values, ranging from $83 \%$ to $99 \%$. However, for organ toxicity and toxicity endpoints (especially for carcinogenicity) (Table 3), probability scores were low and had high variability (51-91\%). This fact could be explained by the quality or adequacy of the database employed for prediction model development. When performing the toxicity endpoint predictions, it could be expected that the best results would be obtained when using a database for model construction composed of chemical compounds or substances similar to those to be predicted. In our case, chemical compounds included in the databases used for model construction were probably not similar enough to the degradation products evaluated. For this reason, the authors suggest revising those predicted results by developing more adequate prediction models.

\section{Conclusions}

The application of PEF treatment to juice and smoothie samples produced mycotoxin reduction percentages from 43 to $70 \%$. The same treatment applied to $\mathrm{H}_{2} \mathrm{O}$ samples produced lower reductions showing that matrix constituents may affect PEF results. After the treatment, degradation products created by the loss of structural aminoacidic fragments of the original molecules, such as HyLv, Val, Ile, or Phe, were identified. The Pro Tox-II server assigned a toxicity class I for ENB degradation products while the rest of the degradation products were classified in toxicity classes III and IV. PEF is presented here as a good strategy to mitigate ENs and BEA contents in juice and smoothie samples, underlying the importance of identification of the degradation products and toxicity assessment.

Thermal treatment would increase ENs and BEA thermolability, allowing reduction of almost all mycotoxins. However, as the retention of heat sensitive vitamins in PEF juices is highly desirable, the increase of the voltage during the treatment, as well as the use of high hydrostatic pressure and ultrasound, are good alternatives to be explored in the future.

Author Contributions: H.B., F.J.B., and E.F.: conceptualization, supervision, and writing-review and editing. J.T.: formal analysis and writing-original draft. N.P.: formal analysis, investigation, and writing-original draft. All authors have read and agreed to the published version of the manuscript.

Funding: This study was supported by the Spanish Ministry of Economy and Competitiveness AGL 2016-77610R and by the pre-PhD program of University of Valencia "Atracció de Talent" (UV-INV-PREDOC16F1-384781). Moreover, the authors also thank Generalitat Valenciana for the financial support (IDIFEDER/2018/046-Procesos innovadores de extracción y conservación: pulsos eléctricos y fluidos supercríticos) through the European Union ERDF funds (European Regional Development Fund).

Conflicts of Interest: The authors declare no conflict of interest.

\section{References}

1. Pinela, J.; Ferreira, I.C. Nonthermal physical technologies to decontaminate and extend the shelf-life of fruits and vegetables: Trends aiming at quality and safety. Crit. Rev. Food Sci. Nutr. 2017, 57, 2095-2111. [CrossRef]

2. Misra, N.N.; Koubaa, M.; Roohinejad, S.; Juliano, P.; Alpas, H.; Inácio, R.S.; Saraiva, J.A.; Barba, F.J. Landmarks in the historical development of twenty first century food processing technologies. Food Res. Int. 2017, 97,318-339. [CrossRef]

3. Picart-Palmade, L.; Cunault, C.; Chevalier-Lucia, D.; Belleville, M.P.; Marchesseau, S. Potentialities and limits of some non-thermal technologies to improve sustainability of food processing. Front. Nutr. 2019, 5, 130. [CrossRef]

4. Zhang, Z.H.; Wang, L.H.; Zeng, X.A.; Han, Z.; Brennan, C.S. Non-thermal technologies and its current and future application in the food industry: A review. Int. J. Food Sci. Technol. 2019, 54, 1-13. [CrossRef]

5. Misra, N.N.; Martynenko, A.; Chemat, F.; Paniwnyk, L.; Barba, F.J.; Jambrak, A.R. Thermodynamics, transport phenomena, and electrochemistry of external field-assisted nonthermal food technologies. Crit. Rev. Food Sci. Nutr. 2018, 58, 1832-1836. [CrossRef] [PubMed]

6. Gabrić, D.; Barba, F.; Roohinejad, S.; Gharibzahedi, S.M.T.; Radojčin, M.; Putnik, P.; Bursać Kovačević, D. Pulsed electric fields as an alternative to thermal processing for preservation of nutritive and physicochemical properties of beverages: A review. J. Food Process. Eng. 2018, 41, e12638. [CrossRef] 
7. Barba, F.J.; Parniakov, O.; Pereira, S.A.; Wiktor, A.; Grimi, N.; Boussetta, N.; Saraiva, J.A.; Raso, J.; Martin-Belloso, O.; Witrowa-Rajchert, D.; et al. Current applications and new opportunities for the use of pulsed electric fields in food science and industry. Food Res. Int. 2015, 77, 773-798. [CrossRef]

8. Salehi, F. Physico-chemical properties of fruit and vegetable juices as affected by pulsed electric field: A review. Int. J. Food Proper. 2020, 23, 1036-1050. [CrossRef]

9. Guo, M.; Jin, T.Z.; Geveke, D.J.; Fan, X.; Sites, J.E.; Wang, L. Evaluation of microbial stability, bioactive compounds, physicochemical properties, and consumer acceptance of pomegranate juice processed in a commercial scale pulsed electric field system. Food Bioprocess Technol. 2014, 7, 2112-2120. [CrossRef]

10. Zhang, Y.; Hou, Y.; Zhang, Y.; Chen, J.; Chen, F.; Liao, X.; Hu, X. Reduction of diazinon and dimethoate in apple juice by pulsed electric field treatment. J. Sci. Food Agric. 2012, 92, 743-750. [CrossRef]

11. Gavahian, M.; Pallares, N.; Al Khawli, F.; Ferrer, E.; Barba, F. Recent advances in the application of innovative food processing technologies for reducing pesticides and mycotoxins in foods. Trends Food Sci. Technol. 2020, in press.

12. Carballo, D.; Pinheiro-Fernandes-Vieira, P.; Tolosa, J.; Font, G.; Berrada, H.; Ferrer, E. Dietary exposure to mycotoxins through fruits juice consumption. Rev. Toxicol. 2018, 35, 2-6.

13. Mallebrera, B.; Prosperini, A.; Font, G.; Ruiz, M.J. In vitro mechanisms of Beauvericin toxicity: A review. Food Chem. Toxicol. 2018, 111, 537-545. [CrossRef] [PubMed]

14. Prosperini, A.; Berrada, H.; Ruiz, M.J.; Caloni, F.; Coccini, T.; Spicer, L.J.; Perego, M.C.; Lafranconi, A. A review of the mycotoxin enniatin B. Front. Public Health 2017, 5, 304. [CrossRef]

15. Karlovsky, P.; Suman, M.; Berthiller, F.; De Meester, J.; Eisenbrand, G.; Perrin, I.; Oswald, I.P.; Speijers, G.; Chiodini, A.; Recker, T.; et al. Impact of food processing and detoxification treatments on mycotoxin contamination. Mycotoxin Res. 2016, 32, 179-205. [CrossRef]

16. Vijayalakshmi, S.; Nadanasabhapathi, S.; Kumar, R.; Kumar, S.; Reddy, R. Effect of combination processing on aflatoxin reduction: Process optimization by response surface methodology. J. Food Process. Preserv. 2017, 41, e13230. [CrossRef]

17. Vijayalakshmi, S.; Nadanasabhapathi, S.; Kumar, R.; Kumar, S.S. Effect of pH and pulsed electric field process parameters on the aflatoxin reduction in model system using response surface methodology. J. Food Sci. Technol. 2018, 55, 868-878. [CrossRef]

18. Cotterill, J.V.; Chaudhry, M.Q.; Matthews, W.; Watkins, R.W. In silico assessment of toxicity of heat-generated food contaminants. Food Chem. Toxicol. 2008, 46, 1905-1918. [CrossRef]

19. Kar, S.; Leszczynski, J. Exploration of computational approaches to predict the toxicity of chemical mixtures. Toxics 2019, 7, 15. [CrossRef]

20. Gozalbes, R.; de Julián-Ortiz, J.V.; Fito-López, C. Métodos computacionales en toxicología predictiva: Aplicación a la reducción de ensayos con animales en el contexto de la legislación comunitaria REACH. Rev. Toxicol. 2014, 31, 157-167.

21. Pallarés, N.; Carballo, D.; Ferrer, E.; Fernández-Franzón, M.; Berrada, H. Mycotoxin Dietary Exposure Assessment through Fruit Juices Consumption in Children and Adult Population. Toxins 2019, 11, 684. [CrossRef] [PubMed]

22. European Commission. Decision 2002/657/EC of 12 August 2002, implementing Council Directive 96/23/EC concerning the performance of analytical methods and the interpretation of results. OJEU L 2002, 221, 8-36.

23. Drwal, M.N.; Banerjee, P.; Dunkel, M.; Wettig, M.R.; Preissner, R. ProTox: A web server for the in silico prediction of rodent oral toxicity. Nucleic Acids Res. 2014, 42, W53-W58. [CrossRef] [PubMed]

24. Banerjee, P.; Eckert, A.O.; Schrey, A.K.; Preissner, R. ProTox-II: A webserver for the prediction of toxicity of chemicals. Nucleic Acids Res. 2018, 46, W257-W263. [CrossRef] [PubMed]

25. United Nations. Globally Harmonized System of Classification and Labelling of Chemicals (GHS) 8th Revised Edition; United Nations: New York, NY, USA; Geneva, Switzerland, 2019. Available online: http://www.unece.org/ fileadmin/DAM/trans/danger/publi/ghs/ghs_rev08/ST-SG-AC10-30-Rev8e.pdf (accessed on 30 October 2019).

26. Serrano, A.B.; Font, G.; Mañes, J.; Ferrer, E. Development a mitigation strategy of enniatins in pasta under home-cooking conditions. LWT-Food Sci. Technol. 2016, 65, 1017-1024. [CrossRef]

27. Tolosa, J.; Font, G.; Mañes, J.; Ferrer, E. Mitigation of enniatins in edible fish tissues by thermal processes and identification of degradation products. Food Chem. Toxicol. 2017, 101, 67-74. [CrossRef] [PubMed]

28. Avsaroglu, M.D.; Bozoglu, F.; Alpas, H.; Largeteau, A.; Demazeau, G. Use of pulsed-high hydrostatic pressure treatment to decrease patulin in apple juice. High Press. Res. 2015, 35, 214-222. [CrossRef] 
29. Hao, H.; Zhou, T.; Koutchma, T.; Wu, F.; Warriner, K. High hydrostatic pressure assisted degradation of patulin in fruit and vegetable juice blends. Food Control 2016, 62, 237-242. [CrossRef]

30. Meca, G.; Ritieni, A.; Mañes, J. Influence of the heat treatment on the degradation of the minor Fusarium mycotoxin beauvericin. Food Control 2012, 28, 13-18. [CrossRef]

31. Serrano, A.B.; Meca, G.; Font, G.; Ferrer, E. Degradation study of enniatins by liquid chromatography-triple quadrupole linear ion trap mass spectrometry. Food Chem. 2013, 141, 4215-4225. [CrossRef]

32. EFSA Panel on Contaminants in the Food Chain (CONTAM). Scientific Opinion on the risks for animal and public health related to the presence of T-2 and HT-2 toxin in food and feed. EFSA J. 2011, 9, 2481. [CrossRef] 